\title{
Chemoprevention for Breast Cancer
}

\author{
Sandhya Pruthi, $\mathrm{MD}^{1}$, Ruth E. Heisey, $\mathrm{MD}^{2}$, and Therese B. Bevers, $\mathrm{MD}^{3}$ \\ ${ }^{1}$ Division of General Internal Medicine, Department of Internal Medicine, Mayo Clinic, Rochester, MN; ${ }^{2}$ Department of \\ Family and Community Medicine, University of Toronto, Women's College Hospital / Princess Margaret Hospital, \\ Toronto, ON, Canada; ${ }^{3}$ Department of Clinical Cancer Prevention, The University of Texas MD Anderson Cancer Center, \\ Houston, TX
}

\begin{abstract}
Background. Many women at increased risk for breast cancer could benefit from preventive therapy. Preventive therapy options for breast cancer risk reduction have expanded in the last few years to include both selective receptor modulators (tamoxifen and raloxifene) and aromatase inhibitors (anastrozole and exemestane).
\end{abstract}

Methods. Risk factors that place women at high risk for breast cancer, as well as risk calculation models appropriate for the selection of candidates for preventive therapy, are presented, followed by a review of current guidelines for chemoprevention and results of chemoprevention trials.

Results. The modified Gail model or Breast Cancer Risk Assessment Tool is the most widely utilized risk assessment calculator to determine eligibility for chemoprevention. Women most likely to benefit from preventive therapy include those at high risk under the age of 50 years and those with atypical hyperplasia. Physician and patient barriers limit widespread acceptance and adherence to preventive therapy.

Conclusions. Published guidelines on chemoprevention for breast cancer have been updated to increase awareness and encourage discussion between patients and their physicians regarding evidence-based studies evaluating the benefits of preventive options for women at increased risk for breast cancer. However, even with increasing awareness and established benefits of preventive therapy, the uptake of chemoprevention has been low, with both

(C) The Author(s) 2015. This article is published with open access at Springerlink.com

First Received: 13 April 2015;

Published Online: 23 July 2015

S. Pruthi, MD

e-mail: pruthi.sandhya@mayo.edu physician and patient barriers identified. It is prudent that these barriers be overcome to enable high-risk women with a favorable risk-to-benefit ratio to be offered chemoprevention to reduce their likelihood of developing hormone receptor-positive breast cancer.

\section{DEFINING BREAST CANCER RISK}

Defining breast cancer risk incorporates knowledge of individual risk factors known to be associated with increased risk. These risk factors are included in various available risk-calculation models to provide a numeric risk that can be used to help quantify the level of individual risk. $^{1}$

Breast cancer risk factors have historically been described as modifiable versus nonmodifiable factors. Modifiable risk factors in general are associated with lifestyle behaviors and exogenous hormone exposure. These include physical inactivity, increased alcohol consumption, obesity, and use of estrogen and progestin therapies, all of which are associated with increasing breast cancer risk. ${ }^{2-5}$ Physicians have an important role in counseling women on the effectiveness of lifestyle modification and avoidance of long-term postmenopausal hormone therapy in the primary prevention of breast cancer. Nonmodifiable risk factors include increasing age, family history, precancerous breast lesions, and reproductive factors (early menarche, lateonset menopause, first live birth after age 30 years, or nulliparity). These risk factors are independently associated with a higher risk of developing breast cancer but it is not known if they are additive for an individual when estimating breast cancer risk.

Breast cancer risk can be categorized as average, high, and very high risk. ${ }^{6}$ In general, a woman having no family history of breast cancer or prior history of a precancerous breast biopsy would be considered at average risk. The 
lifetime risk for developing breast cancer for an averagerisk woman is $12 \%$. The following criteria are most often used to identify women at high risk: (i) first-degree relative with a breast cancer diagnosis before age 50 years; (ii) history of atypical hyperplasia (AH); (iii) 5-year Gail model risk of $\geq 1.7 \%$; (iv) history of lobular carcinoma in situ (LCIS); (v) having received chest radiation between the ages of 10 and 30 years; (vi) increased mammographic breast density; and (vii) International Breast Cancer Intervention Study (IBIS) model (Tyrer-Cuzick) lifetime risk of $\geq 20 \%{ }^{7-12}$ Breast cancer risk factors and the respective absolute or attributable risk of developing breast cancer are described in Table 1.

Women presenting with a strong hereditary predisposition, or known BRCA1 or 2 mutation carriers, are, by definition, considered at very high risk for developing breast cancer. A family history that entails multiple affected relatives with early-onset breast or ovarian cancer over several generations would be an indication to refer to a genetic counselor to discuss the options of genetic testing. The lifetime risk of developing invasive breast cancer for a BRCA mutation carrier is estimated at $40-85 \%{ }^{13}$ Women with a BRCA mutation should be offered bilateral prophylactic mastectomy (BPM) and risk-reducing salpingo-oophorectomy as these are the only risk-reducing strategies shown to be effective in this population. Those not interested in BPM should have enhanced surveillance with annual mammogram and magnetic resonance imaging, and be offered preventive therapy. The evidence of efficacy of preventive therapy in this population is less

TABLE 1 Definition of high risk

\begin{tabular}{|c|c|}
\hline Risk factor & Defining high risk \\
\hline $\begin{array}{l}\text { First-degree family member } \\
\text { diagnosed at }<50 \text { years of } \\
\text { age }\end{array}$ & Twofold risk \\
\hline Atypical hyperplasia & $\begin{array}{l}\text { Cumulative absolute risk is } 30 \% \\
\text { at } 25 \text {-year follow-up }\end{array}$ \\
\hline $\begin{array}{l}\text { Chest radiation between } 10 \text { and } \\
30 \text { years of age }\end{array}$ & $40 \%$ lifetime risk \\
\hline Gail model 5-year risk & Five-year risk $\geq 1.7 \%$ \\
\hline $\begin{array}{l}\text { Breast density (BI-RADS, D3 or } \\
\text { D4) }\end{array}$ & $\begin{array}{l}\text { Women with extremely dense } \\
\text { breasts have a twofold } \\
\text { increased risk compared with } \\
\text { average women }\end{array}$ \\
\hline Lobular carcinoma in situ & $25 \%$ lifetime risk \\
\hline $\begin{array}{l}\text { International Breast Cancer } \\
\text { Intervention Study model } \\
\text { (Tyrer-Cuzick model) life-time } \\
\text { risk }\end{array}$ & $\geq 20 \%$ lifetime risk \\
\hline
\end{tabular}

$B I-R A D S$ Breast Imaging Reporting and Data System, D3 the breast tissue is heterogeneously dense, $D 4$ the breast tissue is extremely dense compelling. ${ }^{14,15}$ Although there is no evidence to support BPM in women who have had thoracic radiation, there is preclinical evidence that tamoxifen decreases the incidence of radiation-induced breast cancer. ${ }^{16,17}$

Several complementary risk assessment and calculation tools are available to assist physicians with making decisions regarding preventive therapy, and individualizing risks. These tools incorporate most of the breast cancer risk factors described above and are easily available to the physician at the point of care. When counseling women about preventive therapy, it is recommended that physicians use a shared decision-making approach with women at high or very high risk as they are most likely to benefit from risk-reduction options. ${ }^{18,19}$ Women with a history of prior chest-wall radiation age $<30$ years, or women with a history of LCIS, are considered to be high enough risk to be considered for preventive therapy [National Comprehensive Cancer Network (NCCN) guidelines version 1.2014 Breast Cancer Risk Reduction]. Other women can be assessed for suitability by using a risk assessment tool.

\section{DETERMINING ELIGIBILITY FOR PREVENTIVE THERAPY/RISK ASSESSMENT TOOLS}

The American Society of Clinical Oncology, the NCCN, the Canadian Task Force on Preventive Health Care, and the US Preventive Services Task Force (USPSTF) advise counseling women $\geq 35$ years of age who are at increased risk for breast cancer regarding available medications to reduce their risk and to offer medication to women at low risk of medication-related side effects (USPSTF B recommendation). ${ }^{20-23}$ The Gail model risk calculator is the most widely utilized tool to identify candidates suitable for chemoprevention. ${ }^{9,24-26}$ The original validated Gail model was updated and modified to become the Breast Cancer Risk Assessment Tool (BCRAT) by the National Cancer Institute and the National Surgical Adjuvant Breast and Bowel Project (NSABP) Biostatistics Center. ${ }^{27}$ The BCRAT includes the following breast cancer risk factors: current age, reproductive history (age at menarche, age at first live birth), history of prior breast disease (number of previous breast biopsies and history of AH), and family history (number of first-degree relatives with breast cancer), with age being the most heavily weighted risk factor. ${ }^{27}$

This model does not include the age of onset of breast cancer in family members, paternal family history, or any family history of ovarian cancer. It is suitable for women $\geq 35$ years of age with no history of ductal carcinoma in situ (DCIS) or LCIS, no prior history of thoracic radiation, and without a strong family history of breast cancer or ovarian cancer suggestive of a genetic predisposition. The model was updated in 2008 to provide adjusted estimates for African American women derived from the 
Women's Contraceptive and Reproductive Experiences (CARE) Study and from Surveillance, Epidemiology and End Results (SEER) data, and in 2011 to include Asian and Pacific Islander women using data from the Asian American Breast Cancer Study (AABCS) combined with the SEER database. ${ }^{25,28}$

Any woman with a 5-year risk of $\geq 1.7 \%$ determined by using the Gail model can be considered for preventive therapy. This is the risk estimate utilized for the major breast cancer prevention trials and supported by NCCN guidelines. ${ }^{21}$ Based on risk-benefit tables developed by Freedman et al. ${ }^{29}$ the USPSTF concludes that, in general, women with an estimated 5-year breast cancer risk of $\geq 3 \%$ are likely to have more benefit than harm from using a selective estrogen receptor modulator (SERM) as chemoprevention, although the balance depends on age, ethnicity, the medication used, and whether or not the patient has a uterus. ${ }^{23}$

In general, women with a history of $\mathrm{AH}$, or women under the age of 50 years, are more likely to benefit from preventive therapy. This is based on the Breast Cancer Prevention Trial (BCPT) data subgroup analysis that demonstrated a significant $86 \%$ risk reduction for women with AH. Furthermore, the evidence supports that women under the age of 50 years are far less likely to incur the harms of therapy seen in women 50 years of age or older. $^{30,31}$ Conversely, in many older women the harms of preventive therapy far outweigh the benefits as their risk of adverse effects is greater.

The NCCN Breast Cancer Risk Reduction Panel has adopted the $1.7 \%$ or greater 5-year actuarial breast cancer risk defined by the modified Gail model as the risk threshold for discussion of chemoprevention. This is consistent with eligibility criteria utilized in the NSABP BCPT and the Study of Tamoxifen and Raloxifene (STAR). ${ }^{30-33}$ Another risk calculation model commonly used is the IBIS or TyrerCuzick model. ${ }^{11}$ It includes BRCA status, height, weight, hormone replacement therapy (HRT) use, age at first live birth, age of onset of cancers in relatives, the presence of ovarian cancer, and second- and third-generation family history on the maternal and paternal side. It is more complex, less accessible to primary care providers, and currently utilized mainly to determine eligibility for enhanced screening with MRI, in addition to mammography, in women with a lifetime risk of breast cancer $\geq 20 \%$.

The recently updated American Society of Clinical Oncology guideline on the use of pharmacological interventions for breast cancer risk reduction states that the risk for breast cancer may be determined by the aforementioned BCRAT tool "or other validated models including TyrerCuzick". ${ }^{20}$

In a head-to-head comparison of the BCRAT and the IBIS model looking at the absolute 10-year risk of breast cancer, the IBIS model showed better discrimination (area under the curve [AUC] for IBIS $69.5 \%, 95 \%$ CI 63.8-75.2 versus AUC for BRCAT $63.2 \%, 95 \%$ CI 57.6-68.9). ${ }^{34}$

There is no validated model that accounts for breast density, yet it is hoped that one might be developed in the future that will include breast density and be capable of effectively identifying women suitable for both enhanced screening and chemoprevention. ${ }^{35}$

\section{PREVENTIVE THERAPY}

Tamoxifen and raloxifene, both SERMs, as well as two aromatase inhibitors (AIs), exemestane and anastrozole, have been shown in randomized controlled trials to significantly reduce breast cancer incidence in women at increased risk of the disease. ${ }^{30-33,36,37}$ The SERMs are US FDA approved for this indication in postmenopausal women, although only tamoxifen has been studied and received an indication for breast cancer risk reduction in premenopausal women. The FDA has not approved either of these two AIs for breast cancer risk reduction, and their use in the US is considered off-label. There are a paucity of data on the effectiveness of preventive therapy in women with a history of chest-wall radiation. ${ }^{38}$

\section{Tamoxifen and Raloxifene}

In the landmark BCPT, tamoxifen reduced the risk of breast cancer in both pre- and postmenopausal women at increased risk of the disease by approximately one-half (relative risk [RR] 0.51; $95 \%$ CI 0.39-0.66). Women with AH had a highly significant $86 \%$ breast cancer risk reduction (RR 0.14; $95 \%$ CI 0.03-0.47), whereas women with LCIS, due to the small sample size, had a nonstatistically significant reduction of $56 \%$ (RR $0.44 ; 95 \%$ CI $0.16-1.06) .{ }^{30}$ Women under the age of 50 years obtained comparable breast cancer risk reduction to women 50 years of age and older. In the 7-year follow-up analysis, the benefits of tamoxifen were shown to persist in women at increased risk of the disease, even after stopping therapy, with a reduction in breast cancer risk of $43 \%$ (RR 0.57 ; $95 \%$ CI 0.46-0.70). Risk remained decreased by $75 \%$ (RR 0.25; $95 \%$ CI 0.10-0.52) in women with $\mathrm{AH}$, while women with LCIS continued to have a nonstatistically significant risk reduction, now $46 \%$ (RR $0.54 ; 95 \%$ CI $0.27-1.02) .{ }^{31}$ An updated analysis of the European IBIS-I trial has demonstrated that tamoxifen continues to reduce breast cancer risk at a median of 16 years of follow-up (HR $0.71 ; 95 \%$ CI $0.60-0.83$ ). The risk of developing breast cancer was similar between years 0-10 (HR 0.72; $95 \%$ CI 0.59-0.88) and after 10 years (HR 0.69; $95 \%$ CI $0.53-0.91){ }^{14}$ 
Tamoxifen is associated with an increased risk of endometrial cancer (RR 2.53; $95 \%$ CI 1.35-4.97; absolute annual risk per 1000: placebo 0.91 vs. tamoxifen 2.30), venous thromboembolic events, including stroke (RR 1.59; $95 \%$ CI 0.93-2.77; absolute annual risk per 1000: placebo 0.92 vs. tamoxifen 1.45), pulmonary embolus (RR 3.01; $95 \%$ CI 1.15-9.27; absolute annual risk per 1000: placebo 0.23 vs. tamoxifen 0.69 ), deep vein thrombosis (RR 1.60; $95 \%$ CI 0.91-2.86; absolute annual risk per 1000: placebo 0.84 vs. tamoxifen 1.34), cataract development (RR 1.14; $95 \%$ CI 1.01-1.29; absolute annual risk per 1000: placebo 21.72 vs. tamoxifen 24.82), and the need for cataract surgeries (RR 1.57; $95 \%$ CI 1.16-2.14; absolute annual risk per 1000: placebo 3.00 vs. tamoxifen 4.72). ${ }^{30}$ These risks were not significantly different in the 2010 analysis. The serious risks were not significantly increased in women under the age of 50 years, thus identifying a population of women who obtain significant risk reduction benefits without incurring serious harm. Common side effects reported included bothersome hot flashes and vaginal discharge.

The STAR demonstrated that raloxifene was equivalent to tamoxifen in reducing breast cancer risk for postmenopausal women at increased risk of the disease while on therapy. ${ }^{32}$ In the 2010 updated analysis, with a median follow-up of 81 months, benefits with tamoxifen were greater, while the risks were lower with raloxifene. Raloxifene retained $76 \%$ of the effectiveness of tamoxifen in preventing invasive disease. Raloxifene was not associated with an increased risk of uterine cancer risk and has a slightly lower risk of venous thromboembolic events than tamoxifen. ${ }^{33}$ Raloxifene is associated with hot flashes, night sweats, vaginal dryness, and weight gain.

\section{Aromatase Inhibitors}

In the National Cancer Institute of Canada (NCIC) Mammary Prevention 3 (MAP.3) trial, after 35 months of follow-up, exemestane reduced breast cancer risk by $65 \%$ (hazard ratio [HR] 0.35; $95 \%$ CI 0.18-0.70) in high-risk postmenopausal women. ${ }^{36}$ A $53 \%$ reduction in breast cancer risk was seen with anastrozole in the European IBIS-II trial in women at increased risk of breast cancer (HR 0.47; $95 \%$ CI 0.32-0.68). ${ }^{37}$ Data on AIs in women with AH or LCIS are limited. In this subgroup, anastrozole reduced breast cancer risk by $69 \%$ (HR $0.31 ; 95 \%$ CI $0.12-0.84),{ }^{37}$ whereas exemestane produced a nonsignificant reduction in the risk of breast cancer by $64 \%$ (HR 0.36 ; $95 \%$ CI $0.11-1.12) .{ }^{36}$ It is important to note that these analyses are in a very small number of women, limiting the ability to assess the effectiveness of therapies in women with LCIS or AH.
Neither exemestane nor anastrozole were associated with an increased risk of thromboembolic or cardiovascular events, or other cancers. In the MAP. 3 trial, although shortterm use of exemestane was shown to worsen age-related bone loss in spite of calcium and vitamin D supplementation, long-term follow-up will be needed to assess the effect on fracture risk in a prevention population. ${ }^{39}$ The side effects of exemestane, including vasomotor, sexual, and musculoskeletal symptoms, had limited impact on quality of life. ${ }^{40}$ In addition to vasomotor symptoms, musculoskeletal events (arthralgias, joint stiffness, carpal tunnel syndrome) were more common in the anastrozole arm. ${ }^{37}$

Although it is important that high-risk women be considered for chemoprevention, several barriers have been identified that impact uptake, compliance, and adherence. These include fear of possible side effects of the antiestrogen therapies, specifically thromboembolic events and an endometrial cancer risk, which may be perceived as outweighing the potential benefits of the pharmacologic therapy on reducing the incidence of breast cancer. ${ }^{41-44}$ Furthermore, it is becoming increasingly evident that physicians are encountering barriers to prescribing pharmacologic therapies, including lack of time to effectively counsel patients about available options, knowledge gaps about the risks and benefits of the medications, and challenges with identifying eligible women with a favorable risk-to-benefit ratio who will benefit from the pharmacologic therapy to reduce breast cancer risk. ${ }^{45,46}$

\section{CONCLUSIONS}

Physicians are strongly encouraged to assess breast cancer risk and appropriately identify high-risk women with a positive risk-benefit ratio eligible for chemoprevention. Communication of the risks and benefits of SERMs and AIs as preventive therapies and shared decision-making approaches are critical to patient uptake and adherence. More widespread utilization of these agents can reduce the incidence of estrogen receptor (ER)-positive breast cancer but will have no impact on ER-negative breast cancer. Future opportunities for breast cancer risk reduction should target hormone-negative, especially triple-negative, breast cancer.

DISCLOSURE Sandhya Pruthi, Ruth E. Heisey, and Therese B. Bevers declare they have nothing to disclose.

OPEN ACCESS This article is distributed under the terms of the Creative Commons Attribution 4.0 International License (http:// creativecommons.org/licenses/by/4.0/), which permits unrestricted use, distribution, and reproduction in any medium, provided you give appropriate credit to the original author(s) and the source, provide a link to the Creative Commons license, and indicate if changes were made. 


\section{REFERENCES}

1. Cummings SR, Tice JA, Bauer S, et al. Prevention of breast cancer in postmenopausal women: approaches to estimating and reducing risk. J Natl Cancer Inst. 2009;101:384-98.

2. Key J, Hodgson S, Omar RZ, et al. Meta-analysis of studies of alcohol and breast cancer with consideration of the methodological issues. Cancer Causes Control. 2006;17:759-70.

3. Eliassen AH, Colditz GA, Rosner B, Willett WC, Hankinson SE. Adult weight change and risk of postmenopausal breast cancer. JAMA. 2006;296:193-201.

4. Lahmann PH, Friedenreich C, Schuit AJ, et al. Physical activity and breast cancer risk: the European Prospective Investigation into Cancer and Nutrition. Cancer Epidemiol Biomarkers Prev. 2007; 16:36-42.

5. Missmer SA, Eliassen AH, Barbieri RL, Hankinson SE. Endogenous estrogen, androgen, and progesterone concentrations and breast cancer risk among postmenopausal women. $J$ Natl Cancer Inst. 2004;96:1856-65.

6. Pruthi S, Heisey R, Bevers T. Personalized assessment and management of women at risk for breast cancer in North America. Womens Health (Lond Engl). 2015;11:213-24.

7. Hartmann LC, Degnim AC, Santen RJ, Dupont WD, Ghosh K. Atypical hyperplasia of the breast-risk assessment and management options. N Engl J Med. 2015;372:78-89.

8. Clemons M, Loijens L, Goss P. Breast cancer risk following irradiation for Hodgkin's disease. Cancer Treat Rev. 2000;26:291-302.

9. Gail MH, Brinton LA, Byar DP, et al. Projecting individualized probabilities of developing breast cancer for white females who are being examined annually. J Natl Cancer Inst. 1989;81: 1879-86.

10. Page DL, Kidd TE Jr, Dupont WD, Simpson JF, Rogers LW. Lobular neoplasia of the breast: higher risk for subsequent invasive cancer predicted by more extensive disease. Hum Pathol. 1991;22:1232-9.

11. Tyrer J, Duffy SW, Cuzick J. A breast cancer prediction model incorporating familial and personal risk factors [published erratum appears in Stat Med. 2005;24(1):156]. Stat Med. 2004;23: 1111-30.

12. Freer PE. Mammographic breast density: impact on breast cancer risk and implications for screening. Radiographics. 2015;35:302-15.

13. Chen S, Parmigiani G. Meta-analysis of BRCA1 and BRCA2 penetrance. J Clin Oncol. 2007;25:1329-33.

14. Cuzick J, Sestak I, Cawthorn S, et al. Tamoxifen for prevention of breast cancer: extended long-term follow-up of the IBIS-I breast cancer prevention trial. Lancet Oncol. 2015;16:67-75.

15. King MC, Wieand S, Hale K, et al. Tamoxifen and breast cancer incidence among women with inherited mutations in BRCA1 and BRCA2: National Surgical Adjuvant Breast and Bowel Project (NSABP-P1) Breast Cancer Prevention Trial. JAMA. 2001;286: 2251-6.

16. Peterson NC, Servinsky MD, Christian A, et al. Tamoxifen resistance and Her2/neu expression in an aged, irradiated rat breast carcinoma model. Carcinogenesis. 2005;26:1542-52.

17. Boucher AA, Blaes AH. Prophylactic mastectomy: a treatment alternative for Hodgkin survivors? Clin Breast Cancer. 2013;13: 307-8.

18. Ropka ME, Keim J, Philbrick JT. Patient decisions about breast cancer chemoprevention: a systematic review and meta-analysis. J Clin Oncol. 2010;28:3090-5.

19. Ozanne EM, Wittenberg E, Garber JE, Weeks JC. Breast cancer prevention: patient decision making and risk communication in the high risk setting. Breast J. 2010;16:38-47.

20. Visvanathan K, Hurley P, Bantug E, et al. Use of pharmacologic interventions for breast cancer risk reduction: American Society of Clinical Oncology clinical practice guideline. J Clin Oncol. 2013;31:2942-62.

21. National Comprehensive Cancer Network. (2014). The NCCN Clinical Practice Guidelines in Oncology (NCCN Guidelines ${ }^{\circledR}$ ) Breast Cancer Risk Reduction (version 1.2014). www.NCCN.org. Accessed 24 Mar 2015.

22. Levine M, Moutquin JM, Walton R, Feightner J. Chemoprevention of breast cancer. A joint guideline from the Canadian Task Force on Preventive Health Care and the Canadian Breast Cancer Initiative's Steering Committee on Clinical Practice Guidelines for the Care and Treatment of Breast Cancer. CMAJ. 2001;164:1681-90.

23. Moyer VA. Medications to decrease the risk for breast cancer in women: recommendations from the U.S. Preventive Services Task Force recommendation statement. Ann Intern Med. 2013;159:698-708.

24. Costantino JP, Gail MH, Pee D, et al. Validation studies for models projecting the risk of invasive and total breast cancer incidence. J Natl Cancer Inst. 1999;91:1541-8.

25. Gail MH, Costantino JP, Pee D, et al. Projecting individualized absolute invasive breast cancer risk in African American women. J Natl Cancer Inst. 2007;99:1782-92.

26. Rockhill B, Spiegelman D, Byrne C, Hunter DJ, Colditz GA. Validation of the Gail et al. model of breast cancer risk prediction and implications for chemoprevention. J Natl Cancer Inst. 2001;93:358-66.

27. National Cancer Institute. Breast Cancer Risk Assessment Tool. Last updated 16 May 2011. www.cancer.gov/bcrisktool. Accessed 22 Mar 2015.

28. Matsuno RK, Costantino JP, Ziegler RG, et al. Projecting individualized absolute invasive breast cancer risk in Asian and Pacific Islander American women. J Natl Cancer Inst. 2011;103:951-61.

29. Freedman AN, Yu B, Gail MH, et al. Benefit/risk assessment for breast cancer chemoprevention with raloxifene or tamoxifen for women age 50 years or older. J Clin Oncol. 2011;29:2327-33.

30. Fisher B, Costantino JP, Wickerham DL, et al. Tamoxifen for prevention of breast cancer: report of the National Surgical Adjuvant Breast and Bowel Project P-1 study. J Natl Cancer Inst. 1998;90:1371-88.

31. Fisher B, Costantino JP, Wickerham DL, et al. Tamoxifen for the prevention of breast cancer: current status of the National Surgical Adjuvant Breast and Bowel Project P-1 study. J Natl Cancer Inst. 2005;97:1652-62.

32. Vogel VG, Costantino JP, Wickerham DL, et al. Effects of tamoxifen vs raloxifene on the risk of developing invasive breast cancer and other disease outcomes: the NSABP Study of Tamoxifen and Raloxifene (STAR) P-2 trial [published errata appear in in JAMA. 2006;296(25):2926, and JAMA. 2007;298(9): 973]. JAMA. 2006;295:2727-41.

33. Vogel VG, Costantino JP, Wickerham DL, et al. Update of the National Surgical Adjuvant Breast and Bowel Project Study of Tamoxifen and Raloxifene (STAR) P-2 trial: preventing breast cancer. Cancer Prev Res (Phila). 2010;3:696-706.

34. Quante AS, Whittemore AS, Shriver T, Strauch K, Terry MB. Breast cancer risk assessment across the risk continuum: genetic and nongenetic risk factors contributing to differential model performance. Breast Cancer Res. 2012;14:R144.

35. Amir E, Freedman OC, Seruga B, Evans DG. Assessing women at high risk of breast cancer: a review of risk assessment models. J Natl Cancer Inst. 2010;102:680-91.

36. Goss PE, Ingle JN, Ales-Martinez JE, et al. Exemestane for breast-cancer prevention in postmenopausal women [published erratum appears in N Engl J Med. 2011;365(14):1361]. N Engl J Med. 2011;364:2381-91.

37. Cuzick J, Sestak I, Forbes JF, et al. Anastrozole for prevention of breast cancer in high-risk postmenopausal women (IBIS-II): an 
international, double-blind, randomised placebo-controlled trial [published erratum appears in Lancet. 2014;383(9922):1040]. Lancet. 2014;383:1041-8.

38. Diller L, Mauch P, Medeiros Nancarrow C, et al. A feasibility study of tamoxifen chemoprevention in Hodgkin's disease (HD) survivors: 8551. J Clin Oncol. 2004;22:811s.

39. Cheung AM, Tile L, Cardew S, et al. Bone density and structure in healthy postmenopausal women treated with exemestane for the primary prevention of breast cancer: a nested substudy of the MAP.3 randomised controlled trial. Lancet Oncol. 2012;13:275-84.

40. Maunsell E, Goss PE, Chlebowski RT, et al. Quality of life in MAP.3 (Mammary Prevention 3): a randomized, placebo-controlled trial evaluating exemestane for prevention of breast cancer. J Clin Oncol. 2014;32:1427-36.

41. Waters EA, McNeel TS, Stevens WM, Freedman AN. Use of tamoxifen and raloxifene for breast cancer chemoprevention in 2010. Breast Cancer Res Treat. 2012;134:875-80.
42. Port ER, Montgomery LL, Heerdt AS, Borgen PI. Patient reluctance toward tamoxifen use for breast cancer primary prevention. Ann Surg Oncol. 2001;8:580-5.

43. Bober SL, Hoke LA, Duda RB, Regan MM, Tung NM. Decisionmaking about tamoxifen in women at high risk for breast cancer: clinical and psychological factors. J Clin Oncol. 2004;22:4951-7.

44. Donnelly LS, Evans DG, Wiseman J, et al. Uptake of tamoxifen in consecutive premenopausal women under surveillance in a high-risk breast cancer clinic. Br J Cancer. 2014;110:1681-7.

45. Kaplan CP, Haas JS, Perez-Stable EJ, Des Jarlais G, Gregorich SE. Factors affecting breast cancer risk reduction practices among California physicians. Prev Med. 2005;41:7-15.

46. Jordan VC. Tamoxifen or raloxifene for breast cancer chemoprevention: a tale of two choices-point. Cancer Epidemiol Biomarkers Prev. 2007;16:2207-9. 